\title{
DETECTION OF ALLELIC VARIANTS OF THE POLE AND POLD1 GENES IN COLORECTAL CANCER PATIENTS
}

\author{
Pätzold LA, Bērziṇa D, Daneberga Z, Gardovskis J, Miklaševičs E*
}

*Corresponding Author: Professor Dr. Edvīns Miklaševičs, Institute of Oncology, Riga Stradiňš University, Dzirciema iela 16, Riga LV1007, Latvia. Tel: +371-6770-4028. Fax: +371-6706-9545. E-mail: edvins. miklasevics@rsu.lv

\begin{abstract}
Incidence of colorectal cancer is high worldwide and it mostly occurs as an accumulation of environmental factors and genetic alterations. Hereditary colorectal cancer can develop as a part of a hereditary syndrome. There is a suspected correlation between colorectal cancer and allelic variants of the POLE and $P O L D 1$ genes. The aim of the present study was to look for associations between the allelic variants in the POLE and POLD1 genes and colorectal cancer. One thousand, seven hundred and forty-nine DNA samples from colorectal cancer patients were collected from 2002 to 2013. Samples were divided in three groups: hereditary colorectal cancer patients, patients with different hereditary cancer syndromes in their families and patients with no cancer history in their families. The DNA samples were screened for allelic variants of POLE rs483352909 and POLD1 rs39751463 using denaturing high performance liquid chromatography (DHPLC). All patients were negative for allelic variants rs483352909 of the POLE gene and rs397514632 of the POLD1 gene. One allelic variant rs 373243003 in the POLE gene and one novel duplication of four nucleotides at the excision site between intron and exon (c.1384-5dupCCTA) in the POLD1 gene, was found. We could not detect or confirm the connection between the genetic variants in the POLD1 and POLE genes and colorectal cancer patients, but we detected a novel genetic variant with an unknown significance.
\end{abstract}

Keywords: Colorectal cancer; denaturing high performance liquid chromatography (DHPLC); POLD1 gene; POLE gene.

\footnotetext{
Institute of Oncology, Riga Stradiņš University, Riga, Latvia
}

\section{INTRODUCTION}

In 2012, the incidence of new colorectal cancer cases were 3421 per 100,000 and mortality 150 per 100,000 individuals of both sexes in Europe [1]. Colorectal cancer is caused by an accumulation of environmental factors and genetic alterations. Hereditary colorectal cancer can develop as a part of a hereditary syndrome. Familial adenomatous polyposis (FAP) develops by allelic variations in the $A P C$ gene causing polyposis and early development of colorectal cancer. The MLH1, MSH2 or MSH6 genes are responsible for the mismatch repair (MMR) system and alterations in these can lead to hereditary nonpolyposis colorectal cancer (HNPCC) or Lynch syndrome. Allelic variants of these genes cover only part of hereditary colo-rectal cancer cases. There is a suspected correlation between colorectal cancer and allelic variants of the POLE and POLD1 genes [2,3]. Replication of the DNA is mostly carried out by three major polymerases, polymerase $\alpha$ (Pol $\alpha$ ), polymerase $\delta(\operatorname{Pol} \delta$ ) and polymerase $\varepsilon(\operatorname{Pol} \varepsilon)$ that are coded by POLA1, POLD1 and POLE genes, respectively. Fidelity of the polymerases, proofreading and the MMR system follow each other to ensure the most accurate replication. It is a highly accurate process and only an error rate of one genetic variant per DNA replication, is observed [4]. Errors during replication are usually corrected by excision of the mismatched nucleotide and reinsertion of the correct base. Ninety to $99.9 \%$ of base-base mismatches are corrected by exonuclease activity of the polymerase itself (Pol $\delta$, Pol $\varepsilon$ ) or by other exonucleases [5]. Occasional errors of proofreading are at last corrected by the MMR system. Pol $\varepsilon$ has a proofreading function due to the POLE domain enabling correction of mistakes caused by the replication process. Those mistakes are caused by either the polymerase itself or polymerase $\alpha$ that has no proofreading capacity [2]. Exonuclease-proficient Pol $\varepsilon$ has 
an error rate of $6.5 \times 10^{-4}$, whereas exonuclease-deficient Pol $\varepsilon$ has an error rate $46 \times 10^{-4}$ [4]. Polymerase $\delta$ and its catalytic and exonuclease subunit POLD1 have the function of bulk replication of DNA of the lagging strand. It is the equivalent to the Pol $\varepsilon$ and it also has the ability of proofreading, base excision repair and Okazaki fragment maturation. The exonuclease-proficient Pol $\delta$ has an error rate of $4.5 \times 10^{-6}$, where the exonuclease-deficient Pol $\delta$ has an error rate of $44 \times 10^{-6}$ [6]. Palles et al. [2] confirmed an association between the formation of colorectal adenomas and carcinomas and heterozygous allelic variants of these genes. They found rare missense allelic variants, p.S478N (rs397514632) in the POLD1 gene and p.L424V (rs483352909) in the POLE gene, which caused malfunctions of the exonuclease subunit of Pol $\delta$ and Pol $\varepsilon$ [2]. Jansen et al. [3] described two germline variants in POLE/ $P O L D 1$ and different somatic variants in POLE/POLD1 and $M M R$ genes in HNPCC patients with no known disease causing germline variant in $M M R$ genes. The patients were suspected of carrying HNPCC because of Amsterdam II and Bethesda criteria. They concluded that loss of proofreading might be the cause of faulty MMR and the cause of the tumor formation [3]. Albertson et al. [7] also showed high incidence of sporadic intestinal adenomas and carcinomas in mice with inbred homozygous genetic variants in the POLE gene. Mice with inbred POLD1 genetic variants showed tumor formations of the skin, lungs and thymus [7]. The aim of the present study was to look for associations between the allelic variants in POLE (rs483352909) and POLD1 (rs397514632) and colorectal cancer.

\section{MATERIALS AND METHODS}

Study Group. One thousand, seven hundred and forty-nine DNA samples from colorectal cancer patients were collected from 2002 to 2013. The patients' age at diagnosis ranges from 20 to 93 with a mean age of 67.09 \pm 10.96 . The hereditary colorectal cancer group consisted of 230 patients corresponding to Amsterdam I and II criteria and the Bethesda criteria. The age at diagnosis in this group ranged from 24 to 88 with a mean age of 67.5 . The second group of hereditary cancer syndromes including hereditary breast/ovarian cancer, hereditary endometrial cancer, hereditary stomach cancer and cancer aggregation in the family consisted of 163 patients. The age at diagnosis ranged from 35 to 82 years with a mean age of 67 . The rest of the samples consisted of 1356 patients with no history or unknown history of cancer in the family. The age of diagnosis ranged from 20 to 93 with a mean age of 70 . All samples belonging to the HNPCC group were tested and found negative for pathogenic allelic variants of the MLH1, MSH2 and MSH6 genes. None of those samples had been screened for genetic variants of the POLE or $P O L D 1$ genes before. Informed consent was obtained from all individual participants included in the study.

Methods. DNA was extracted from whole blood using Flexi gene DNA kit (Qiagen GmbH, Hilden, Germany) and polymerase chain reaction (PCR) was done using a novel proofreading polymerase Optimase, from thermophilic Archaeal bacterium manufactured by Transgenomic Inc. (Omaha, NE, USA). The DNA samples were screened for allelic variants of POLE rs483352909 and POLD1 rs397514632. The primers were self-designed and in the case for POLD1, they were designed to exclude a common heterozygous variant in preferred PCR fragment. Primers for POLE rs483352909 were forward 5'-ATG TCC TCC GGG TCT AGC TC-3' and reverse 5'-GGT GCC TGT TAG GAA CTT GC-3'; for POLD1 rs397514632, forward 5'-ACT CTT CAT TCC AGT CCA AG-3', and reverse 5'-TGA TGA TGC TGT GCT GC3 '. The annealing temperature was $58^{\circ} \mathrm{C}$ for $P O L E$ and $59.5^{\circ} \mathrm{C}$ for $P O L D 1$ primers. The elongation time was set to $1 \mathrm{~min}$. as the proofreading polymerase needs approximately twice as long for elongation. After PCR, the samples were denaturated from $95{ }^{\circ} \mathrm{C}$ to $25^{\circ} \mathrm{C}$ ramping $1.5^{\circ} \mathrm{C}$ per min. The PCR products were then analyzed by denaturing high performance liquid chromatography (DHPLC). The DHPLC analysis was performed using the WAVE® system (model 4500; Transgenomic Inc.), which is equipped with DNASep ${ }^{\circledR} H T$ Cartridge, UV Detector (model L-2400), Oven (model L-2310T), autosampler (model L-2200) and pump (model L-2310) (all from Transgenomic Inc.). The DNA was detected using the cartridge in combination with the buffers $\mathrm{A}(0.1 \mathrm{M}$ TEAA aqueous solution) and B (0.1M TEAA aqueous solution with $25.0 \%$ acetonitrile). The conditions for DHPLC were established using Transgenomic Navigator ${ }^{\mathrm{TM}}$ software and adjusted by adding positive and negative controls in each sample set (Figure 1). The DHPLC analysis of one sample with the rapid DNA method takes $3.5 \mathrm{~min}$. The optimal temperature was found by titrating it at $\pm 1.5^{\circ} \mathrm{C}$ and the necessary TEAA and acetonitrile concentrations were calculated according to the constitution of the fragment by the Navigator ${ }^{\mathrm{TM}}$ software. The partial denaturation temperature for POLE was $62^{\circ} \mathrm{C}$ and for POLDI $63{ }^{\circ} \mathrm{C}$. This method was validated and deviations from wildtype curves seen in DHPLC were then analyzed by Sanger sequencing using capillary electrophoresis with ABI PRISM ${ }^{\mathrm{TM}} 3130$ (Applied Biosystems, Foster City, CA, USA) (Figure 2). 


\section{RESULTS}

All screened patients were negative for allelic variant rs483352909 of the POLE gene. Only one sample showed a disturbed chromatogram peak profile. Upon sequencing it was identified as the allelic variant of the POLE gene rs373243003 (c.1227-49C >T) (Figure 3), which is probably benign as the base substitution is in the intron and may not affect the protein synthesis. The allelic variant rs397514632 of the POLD1 gene was not found in any patient. Nevertheless, a duplication of four nucleotides at the excision site between intron and exon (c.1384-5dup CCTA) was found (Figure 4), which may alter the splicing site. The patient carrying this allelic variant of the POLD1 gene was diagnosed with colorectal cancer at the age of 73 . He reported no family history of colorectal cancer but the full pedigree for this family was not available.

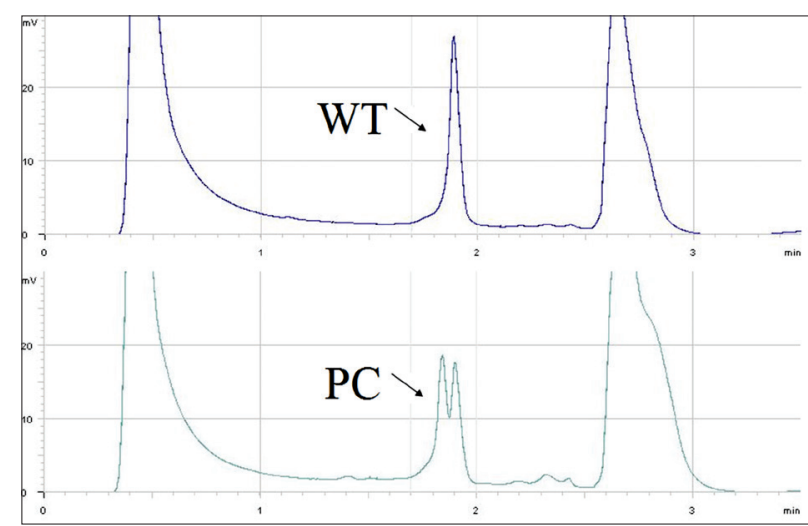

Figure 1. Chromatographic analysis of $P O L D 1$ in negative control DNA sample (WT) and positive control DNA sample (PC).

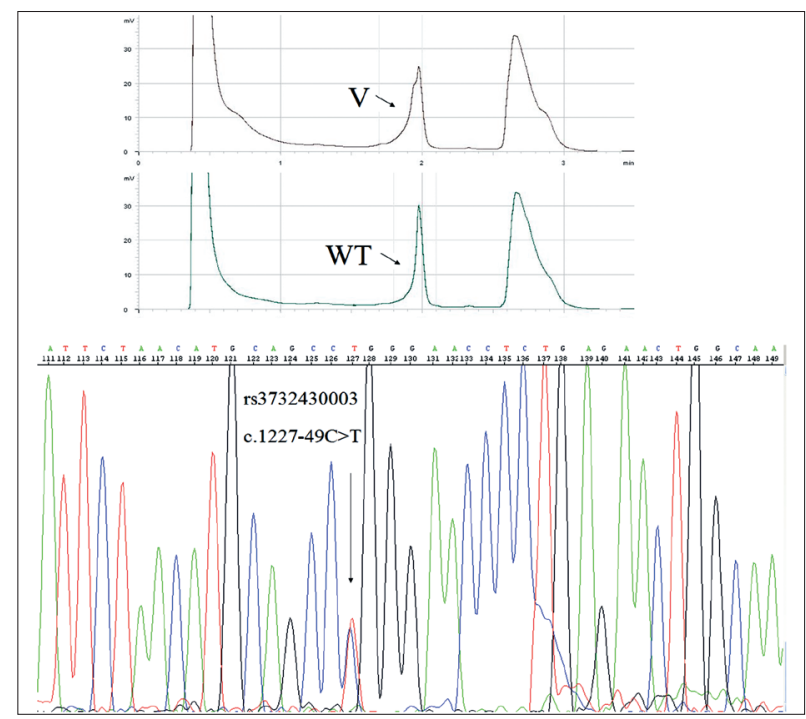

Figure 3. Chromatographic analysis of POLE, positive sample (V) compared to negative control sample (WT) and capillary electrophoresis of positive sample, revealing the POLE c. $1227-49 \mathrm{C}>\mathrm{T}$ variant.

\section{DISCUSSION}

Pol $\varepsilon$ and Pol $\delta$ are two enzymes with a significant protective role in DNA replication with their ability of proofreading. Allelic variants of the POLD1 and POLE genes could alter the function of these enzymes leading to more frequent faulty DNA replication. This can potentially lead to more cases of hereditary colorectal cancer. Our patients did not show the conversion of $\mathrm{S} 478 \mathrm{~N}$ in $P O L D 1$ or L424V in POLE. This could be explained by the difference in patient groups taken for analysis. The Latvian and British populations both have diverse gene pools, which are mostly not overlapping. As our sample size was quite large and the patients come from every region in Latvia, it represented a large portion of the Latvian gene pool. The genetic variability in Latvia is also large due to its history. We especially screened for these two genetic variants of the $P O L E$ and $P O L D 1$ genes in greater expectation of positive results, which would correspond to the previous findings

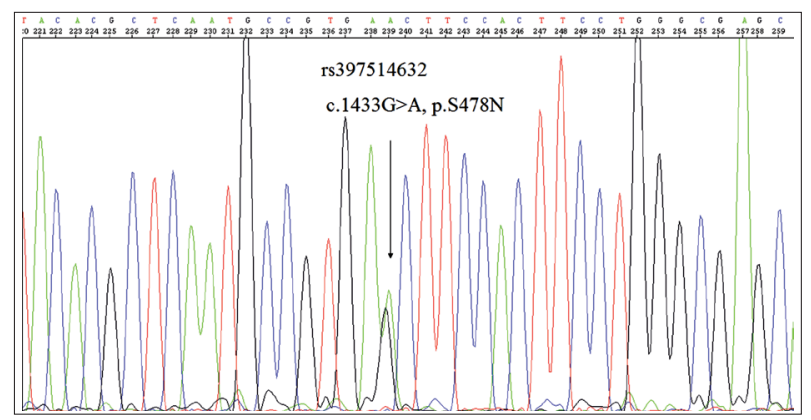

Figure 2. Capillary electrophoresis of $P O L D 1$ positive control DNA sample.

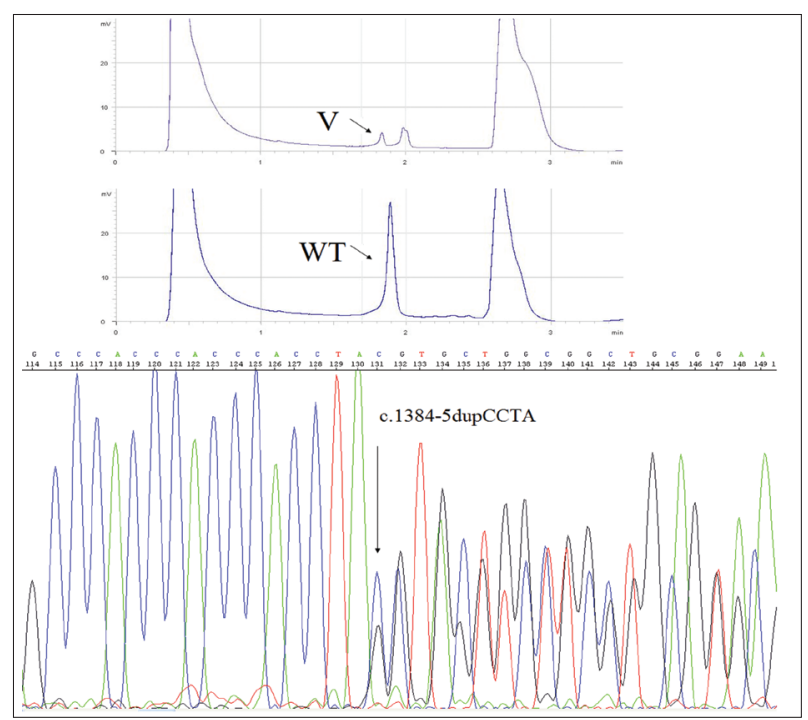

Figure 4. Chromatographic analysis of $P O L D 1$, positive sample (V) compared to negative control sample (WT) and capillary electrophoresis of positive sample, revealing the $P O L D 1 \mathrm{c} .1384-5$ dupCCTA variant. 
in similar studies. There is great theoretical potential to detect genetic variants in other exons of Pol $\delta$ and Pol $\varepsilon$, but this patient group was not screened for genetic variants in other gene regions. In the future we should take a look at those other regions of these two genes, as genetic variation could have great influence on the function of these two enzymes. To diagnose HNPCC or other hereditary cancers, patients need to fulfill criteria that often include detailed information about their patients' family and the history of diseases running in those families. According to Vanags et al. [8], in breast cancer patients, families corresponding to hereditary cancer criteria are larger than families of patients with $B R C A 1$ genetic variants, which do not fit to hereditary cancer criteria due to lack of knowledge about their family history. The same is seen in the case of colorectal cancer patients. Patients diagnosed according to the Amsterdam I criteria are found less often than patients who are diagnosed according to Amsterdam II and Bethesda criteria and proven to carry pathogenic variants in $M M R$ genes [9]. We expected to find, at least in the hereditary colorectal cancer group, pathogenic variants of POLE or POLD1. This would correspond to the findings of Palles et al. [2], and Jansen et al. [3], as this subgroup consists in part of HNPCC patients. On the contrary, we found and sequenced one allelic variant with an unknown significance (c.1384-5dupCCTA) on the POLD1 gene in a male patient, who belongs to the subgroup with an unknown genetic background. This genetic variant has not been described before. A duplication of four nucleotides was found at the 3 ' end of intron 11, which covers the splicing site of intron 11 and exon 12 of this gene. The intron belongs to the U2-type introns, as do $95.0 \%$ of all introns, and it is spliced by major spliceosomes [10]. As this genetic variant is not listed on the National Center for Biotechnology Information (NCBI) single nucleotide polymorphism (SNP) database and has not been published elsewhere, we can only predict the potential effect of this allelic variant on the splicing mechanism. An intron needs to fulfill certain requirements to ensure accurate splicing. Important features are conserved length between the branch point sequence (BPS) and the 3' end and also conserved sequences at the $5^{\prime}$ ' and 3 ' ends of the intron [11,12]. This is important for the binding of the spliceosome, lariat formation and cleavage at the 3' end. Reed [12] showed that long pyrimidine stretches between the BPS and the 3' end enhance the first step of the splicing. However, he also showed that if the distance between the BPS and the 3, end increases or if the sequence is altered, the second step of splicing is significantly slowed down and the efficiency decreases. This could lead to non splicing of the intron and exon. Therefore, the first step of the splicing reaction could be enhanced as the genetic variant c.1384-5dupCCTA has three more pyrimidine bases (CCT) between the BPS and the 3' end. Moreover, the efficiency of the second step of the reaction might decrease, as the length of the sequence between the BPS and the 3' end is increased and also the sequence changed [12]. Thus, we concluded that this genetic variant most probably is clinically significant, as there are possible modifications in the intron-exon splice site. Even though this speculation was supported by molecular biology, the late age of diagnosis of this patient is speaking against it. This is just an assumption because the enzyme generated by this particular DNA alteration needs to be assessed before we can clearly see the effects on splicing. As none of the other samples of our study group showed the same genetic variant in that particular region of the gene, it seems unnecessary to screen healthy control samples for this variation. As our sample size is quite large and the diversity of patients is high, no other result is expected from screening healthy controls. All in all, we could not detect or confirm the connection between the genetic variants in the POLD1 and POLE genes and colorectal cancer patients, but we detected a novel genetic variant with an unknown significance.

\section{ACKNOWLEDGMENTS}

We are grateful to Dr. Claire Palles and Professor Ian Tomlinson (Institute of Cancer and Genomic Sciences, University of Birmingham, Birmingham, UK), for providing us with positive controls of the POLD1 and POLE allelic variants.

Declaration of Interest. The authors report no conflicts of interest. The authors alone are responsible for the content and writing of this article.

\section{Funding}

This study was supported by the State research program "Biomedicine for Public Health (BIOMEDICINE)."

\section{REFERENCES}

1. Ferlay J, Steliarova-Foucher E, Lortet-Tieulent J, Rosso S, Coebergh JW, Comber H, et al. Cancer incidence and mortality patterns in Europe: Estimates for 40 countries in 2012. Eur J Cancer. 2013; 49(6): 1374-1403.

2. Palles C, Cazier JB, Howarth KM, Domingo E, Jones AM, Broderick P, et al. Germline mutations affecting the proofreading domains of POLE and POLD1 
predispose to colorectal adenomas and carcinomas. Nat Genet. 2013; 45(2): 136-144.

3. Jansen AM, van Wezel T, van den Akker BE, Ventayol Garcia M, Ruano D, Tops CM, et al. Combined mismatch repair and POLE/POLD1 defects explain unresolved suspected Lynch syndrome cancers. Eur J Hum Genet. 2016; 24(7): 1089-1092.

4. Korona DA, Lecompte KG, Pursell ZF. The high fidelity and unique error signature of human DNA polymerase epsilon. Nucleic Acids Res. 2011; 39(5): 1763-1773.

5. Kunkel TA. DNA replication fidelity. J Biol Chem. 2004; 279(17): 16895-16898.

6. Schmitt MW, Matsumoto Y, Loeb LA. High fidelity and lesion bypass capability of human DNA polymerase delta. Biochimie. 2009; 91(9): 1163-1172.

7. Albertson TM, Ogawa M, Bugni JM, Hays LE, Chen $\mathrm{Y}$, Wang $\mathrm{Y}$, et al. DNA polymerase $\varepsilon$ and $\delta$ proofreading suppress discrete mutator and cancer phenotypes in mice. Proc Natl Acad Sci USA. 2009; 106(40): 17101-17104.
8. Vanags A, Strumfa I, Gardovskis A, Borosenko V, Abolinšs A, Teibe U, et al. Population screening for hereditary and familial cancer syndromes in Valka district of Latvia. Hered Cancer Clin Pract. 2010; 8(1): 8 .

9. Berzina D, Irmejs A, Kalniete D, Borosenko V, Nakazawa-Miklaševiča M, Rībenieks K, et al. Novel germline MLH1 and MSH2 mutations in Latvian Lynch syndrome families. Exp Oncol. 2012; 34(1): 49-52.

10. Sharp PA, Burge CB. Classification of introns: U2type or U12-type. Cell. 1997; 91(7): 875-879.

11. Patel AA, Steitz JA. Splicing double: Insights from the second spliceosome. Nat Rev Mol Cell Biol. 2003; 4(12): 960-970.

12. Reed R. The organization of 3' splice-site sequences in mammalian introns. Genes Dev. 1989; 3(12B): 2113-2123. 